\title{
Структура зоны проводимости теллурида висмута по данным оптического поглощения
}

\author{
(C) А.Н. Вейс ${ }^{1}$, Л.Н. Лукьянова ${ }^{2, q}$, В.А. Кутасов 2 \\ ${ }^{1}$ Санкт-Петербургский государственный политехнический университет Петра Великого, \\ 195251 Санкт-Петербург, Россия, \\ Физико-технический институт им. А.Ф. Иоффре Российской академии наук, \\ 194021 Санкт-Петербург, Россия \\ "E-mail: lidia.lukyanova@mail.ioffe.ru \\ (Получена 27 декабря 2016 г. Принята к печати 12 января 2017 г.)
}

\begin{abstract}
Проведены исследования спектральных зависимостей коэффициента оптического поглощения $n$ - $\mathrm{Bi}_{2} \mathrm{Te}_{3}$ в диапазоне 40-300 мэВ в зависимости от концентрации электронов и толщины образцов при комнатной температуре с целью определения параметров дополнительной подзоны в зоне проводимости теллурида висмута и ее возможного влияния на транспорт носителей заряда. Показано, что теллурид висмута является прямозонным полупроводником с дополнительной подзоной в зоне проводимости. Эти данные согласуются с исследованиями квантовых осцилляций в $n-\mathrm{Bi}_{2} \mathrm{Te}_{3}$ в сильных магнитных полях при температурах ниже $20 \mathrm{~K}$.
\end{abstract}

DOI: 10.21883/FTP.2017.07.44630.16

Слоистые полупроводники группы $\mathrm{A}_{2}^{\mathrm{V}} \mathrm{B}_{3}^{\mathrm{VI}}$ широко известны и исследуются более полувека, однако их энергетический спектр до настоящего времени изучен не достаточно. Лишь недавно [1,2] было установлено, что теллурид висмута - наиболее известный материал среди всех соединений указанной группы, а также ряд твердых растворов на его основе, в том числе и неизоморфных $\mathrm{Bi}_{2-x} \mathrm{Sb}_{x} \mathrm{Te}_{3-y-z} \mathrm{Se}_{y} \mathrm{~S}_{z}$, широко используемых в термоэлектричестве, являются прямозонными полупроводниками. Знание параметров энергетического спектра полупроводниковых материалов необходимо для их технического применения. В частности, это позволяет изыскивать пути управления величиной фактора мощности $S_{0}^{2} \sigma\left(S_{0}-\right.$ коэффициент термоэдс, $\sigma$ - электропроводность), определяющего эффективность транспорта носителей заряда. Поэтому исследования, направленные на изучение зонного спектра $\mathrm{Bi}_{2} \mathrm{Te}_{3}$ и твердых растворов на его основе, до сих пор являются актуальными. Цель настоящей работы состоит в том, чтобы получить экспериментальные доказательства существования дополнительной подзоны в зоне проводимости теллурида висмута.

Особенности, связываемые с дополнительной электронной подзоной, неоднократно наблюдались при исследовании квантовых осцилляций в $n-\mathrm{Bi}_{2} \mathrm{Te}_{3}$ [3,4]. Однако до сих пор не получено каких-либо убедительных доказательств ее существования при помощи других экспериментальных методик. Не удалось получить их и посредством исследования спектров оптического отражения в $n-\mathrm{Bi}_{2} \mathrm{Te}_{3}$ [5]. Концентрационная зависимость эффективных масс проводимости $m_{R}$ в нем была описана в рамках обобщенной модели Кейна, без привлечения представлений о существовании в теллуриде висмута подзоны „тяжелых“ электронов. Однако в этих исследованиях был обнаружен иной, и как оказалось впоследствии, очень важный эффект. Сопоставление парамет- ров кейновской модели, полученных в работе [5] при $T=300 \mathrm{~K}$, с данными Кёллера [4], полученными при $T=1.6 \mathrm{~K}$, показало, что при повышении температуры в $n-\mathrm{Bi}_{2} \mathrm{Te}_{3}$ наблюдается резкое изменение величины соотношения между оптической шириной запрещенной зоны $E_{g d}$ и эффективным зазором взаимодействия $E_{g}^{*}$. По данным оптических экспериментов $E_{g}^{*}<E_{g d}$, тогда как у Кёллера $E_{g}^{*}>E_{g d}$. Это, а также очень быстрое возрастание величин $m_{R}$ при увеличении температуры, позволило авторам работы [5] предположить, что существенное влияние на параметры энергетического спектра $\mathrm{Bi}_{2} \mathrm{Te}_{3}$ оказывает обменное взаимодействие электронов [6], обусловливающее смещение дна занятой электронами проводимости зоны вниз по шкале энергий (см. энергетическую схему $\mathrm{Bi}_{2} \mathrm{Te}_{3}$, показанную на рис. 1). Оказалось, что для согласования знаков в соотношении между $E_{g d}$ и $E_{g}^{*}$, полученных при анализе данных работ [4,5], достаточно того, чтобы скорость изменения $E_{g d}$ с ростом $n$ была равна $\delta E_{g d}^{e e}=0.9 \cdot 10^{-2}\left(n / 10^{18}\right)$ эВ $(n-$ концентрация свободных электронов). Смещение дна зоны проводимости вниз, в свою очередь, обусловливает возрастание энергетического зазора $\Delta E_{c}$ между подзонами ,легких“ и „тяжелых“ электронов. Вследствие этого дополнительная подзона зоны проводимости $\mathrm{Bi}_{2} \mathrm{Te}_{3}$ может и не проявиться при исследовании спектров оптического отражения. Поэтому поиск дополнительных доказательств существования подзоны „тяжелых“ электронов в $\mathrm{Bi}_{2} \mathrm{Te}_{3}$ должен производиться при помощи изучения спектров оптического поглощения $\alpha(h v)$.

Учитывая все вышесказанное, можно ожидать, что искомая особенность энергетического спектра $\mathrm{Bi}_{2} \mathrm{Te}_{3}$ проявится в зависимостях $\alpha(h v)$ в виде несимметричных полос дополнительного поглощения, обладающих размытой по шкале энергий красной границей, которая по мере увеличения $n$ будет смещаться в коротковолновую 


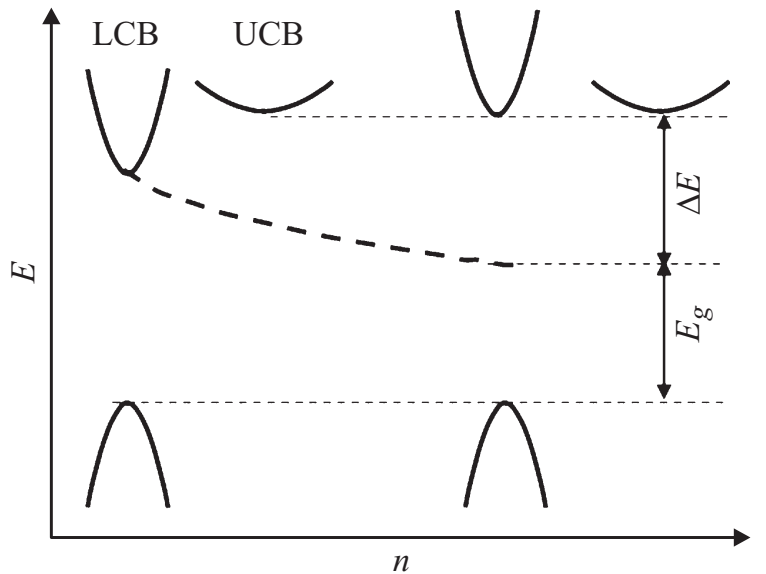

Pис. 1. Схема эволюции зонной структуры $n-\mathrm{Bi}_{2} \mathrm{Te}_{3}$ за счет обменного взаимодействия свободных электронов. Подзона „тяжелых“ дырок не показана. LCB - нижняя зона проводимости, UCB - верхняя зона проводимости.

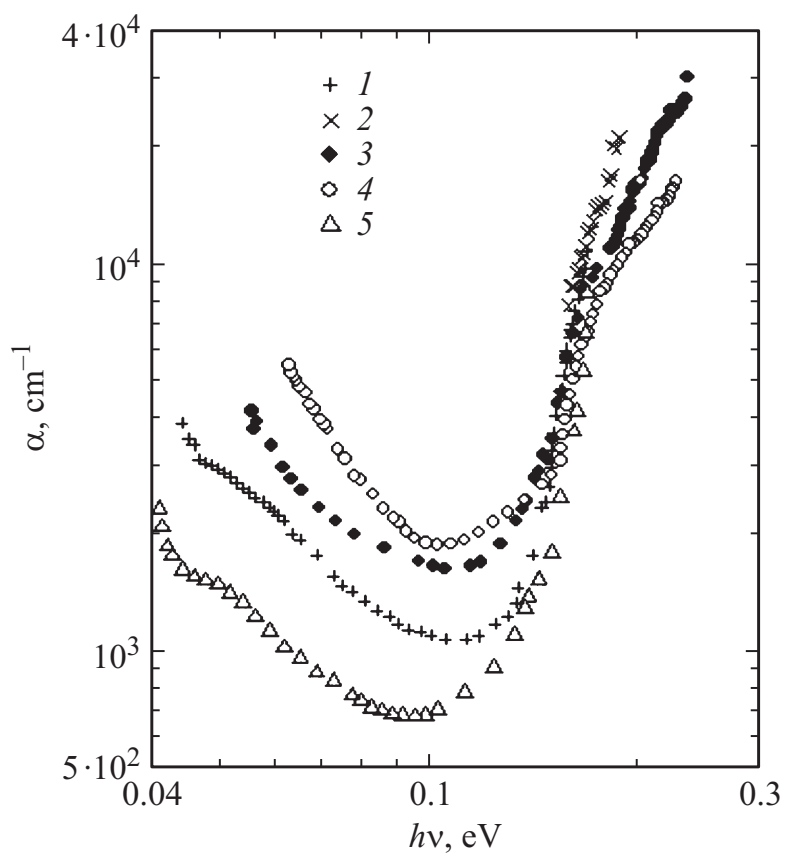

Рис. 2. Спектральные зависимости коэффициента поглощения $\alpha$ в $n$ - $\mathrm{Bi}_{2} \mathrm{Te}_{3} . n, 10^{18} \mathrm{~cm}^{-3}: 1,2,5-2.0,3-4.7,4-6.6$; $d$, мкм: $1,5-4.93,2-3.19,3-2.37,4-2.96 ; T, \mathrm{~K}$ : $1-4-300,5-205$.

область. Если предположение, высказанное авторами [5], верно, то абсолютные величины скорости изменения энергетического зазора $\delta \Delta E_{c}$ и скорости изменения ширины прямой запрещенной зоны $\delta E_{g d}^{e e}$ должны быть равны.

Все исследованные образцы были выращены методом направленной кристаллизации с прецизионной регулировкой на фронте кристаллизации. Необходимые концентрации свободных носителей заряда были получены посредством добавления в шихту избыточного теллура.
Образцы, необходимые для исследования спектров оптического поглощения, были вырезаны из монокристаллических зерен, из которых состоят выращенные слитки. Тонкие образцы выкалывались из зерен, их толщина была определена из положения интерференционных экстремумов в спектрах прозрачности.

Экспериментальные данные настоящей работы показаны на рис. 2. Видно, что спектры оптического поглощения $n-\mathrm{Bi}_{2} \mathrm{Te}_{3}$ обладают достаточно сложной структурой. В их коротковолновой области присутствует край собственного поглощения. Снижение значений $\alpha$ при возрастании концентрации свободных электронов, наблюдаемое в этой области, свидетельствует об увеличении степени вырождения электронного газа. В длинноволновой области всех спектров доминирует поглощение свободными носителями заряда. Однако частотные зависимости $\alpha(h v)$ в ней не в полной мере отвечают степенным зависимостям типа

$$
\alpha_{f c} \propto(h v)^{-k},
$$

характерным для указанного механизма поглощения света, где $\alpha_{f c}-$ коэффициент поглощения свободными электронами. Это свидетельствует о том, что наряду с поглощением свободными носителями заряда в длинноволновой области всех экспериментальных спектров присутствуют дополнительные составляющие коэффициента поглощения $\alpha_{a d d}$.

Эти составляющие были выделены из экспериментальных кривых посредством вычитания экстраполированного по закону (1) поглощения свободными электронами. Для экстраполяции $\alpha_{f c}$ были использованы экспериментальные точки, расположенные вблизи длинноволновой границы экспериментальных спектров, поскольку вклад в суммарное поглощение со стороны свободных электронов там наибольший.

Очевидно, при данном способе экстраполяции зависимостей $\alpha_{f c}(h v)$ могут возникать существенные ошибки в величинах параметра $\mathbf{k}$, присутствующего в формуле (1). Поэтому при построении экстраполяционных прямых в двойном логарифмическом масштабе, соответствующих формуле (1), был проварьирован их наклон в пределах, допускаемых разбросом экспериментальных точек.

Построенные таким образом зависимости $\alpha_{f c}$ были вычтены из экспериментальных кривых, а полученные в результате этой процедуры спектры $\alpha_{a d d(i)}(h v)$ анализировались отдельно. Это позволило найти средние значения энергетического зазора между подзонами зоны проводимости $\left\langle\Delta E_{c}\right\rangle$ в каждом из исследованных образцов и оценить величины их погрешностей с надежностью $s=0.9$.

Некоторые из полученных таким образом результатов, а именно те, анализ которых позволил получить значения $\Delta E_{c(i)}$, наиболее близкие к $\left\langle\Delta E_{c}\right\rangle$, показаны на рис. 3 точками. Данные, представленные на рис. 3, свидетельствуют о том, что полосы $\alpha_{a d d}$ действительно связаны с межподзонными переходами электронов в 


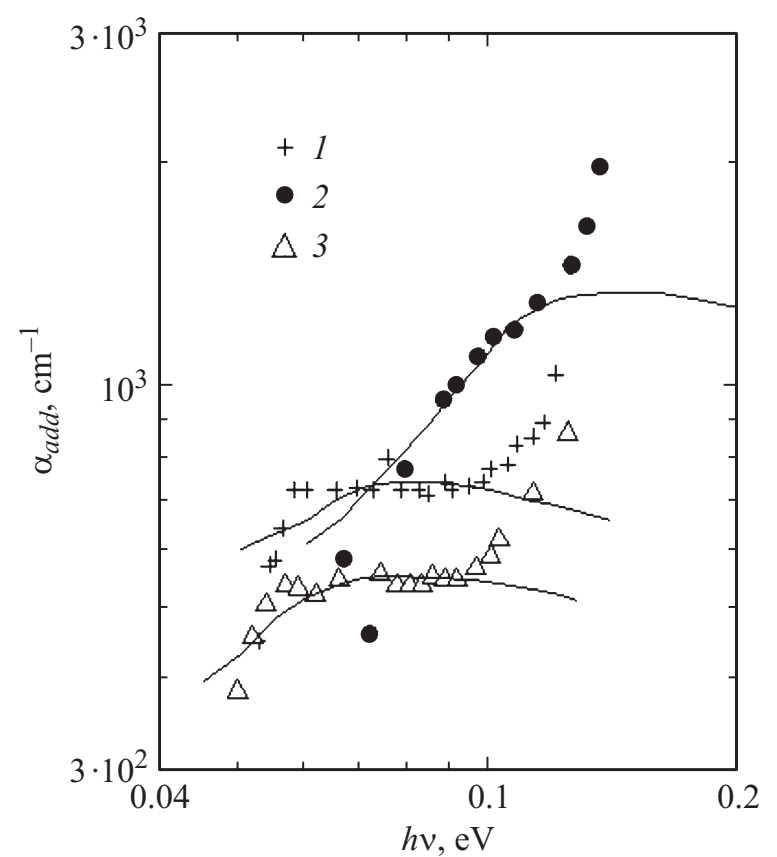

Рис. 3. Спектры $\alpha_{a d d(i)}(h v)$ в $n-\mathrm{Bi}_{2} \mathrm{Te}_{3} . n, 10^{18} \mathrm{~cm}^{-3}: 1,3-2$, $2-4.7$; $d$, мкм: $1,3-4.93,2-2.37 ; T, \mathrm{~K}: 1,2-300$, $3-205$.

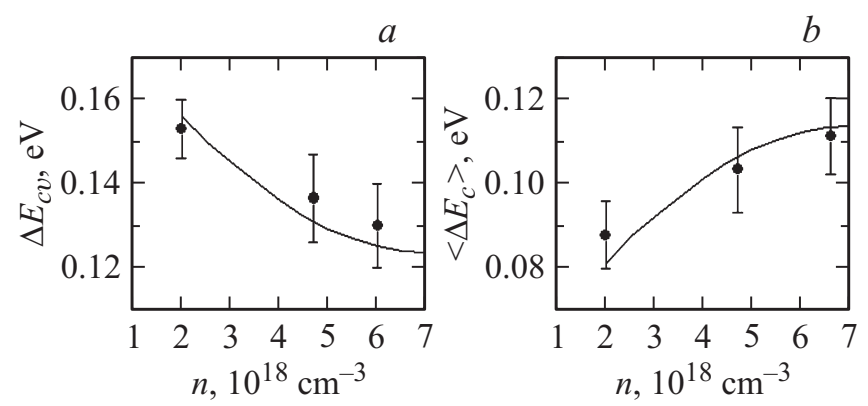

Рис. 4. Концентрационные зависимости щелей $\Delta E_{c v}(a)$ и $\left\langle\Delta E_{c}\right\rangle$ (b) при $T=300 \mathrm{~K}$. Точки - эксперимент, линии расчет, выполненный в соответствии с результатами работы [5].

зоне проводимости теллурида висмута. На это указывает их несимметричная форма и наличие у них лишь одной, красной границы поглощения, размытой по шкале энергий. Не противоречит этому предположению и уменьшение интенсивности полосы дополнительного поглощения при понижении температуры, наблюдаемое в образце с низкой концентрацией электронов. Учитывая сложность фононного спектра теллурида висмута [7], такой эффект в этом материале вполне возможен.

Отметим также, что с ростом концентрации свободных электронов красная граница полос дополнительного поглощения смещается в коротковолновую область спектра. Этот эффект обусловлен обменным взаимодействием электронов. Наоборот, при понижении температуры образца красная граница полос $\alpha_{a d d}$ сдвигается в обратном направлении, в сторону длинных волн.
Это свидетельствует о том, что знак у коэффициента термического изменения энергетической щели между подзонами зоны проводимости положителен.

Величины энергетической щели $\Delta E_{c}$ во всех исследованных образцах были определены при помощи расчета спектров дополнительного поглощения $\alpha_{a d d(i)}(h v)$, выполненного в рамках теории Хаги и Кимуры [8] для переходов I типа. Результаты расчета показаны на рис. 3 линиями, а величины $\left\langle\Delta E_{c}\right\rangle$, определенные при этом, представлены на рис. 4 (при построении этого рисунка данные, полученные для двух образцов с низкой концентрацией электронов, были усреднены).

Для того чтобы показать, что изменение величины $\left\langle E_{c}\right\rangle$ действительно обусловлено электрон-электронным взаимодействием, во всех исследованных образцах была определена величина энергетической щели $\Delta E_{c v}$ между абсолютными экстремумами электронной и дырочной зон. Для этого была использована методика, развитая в работе [9]. Полученные данные также представлены на рис. 4. Видно, что в $n-\mathrm{Bi}_{2} \mathrm{Te}_{3}$ величины $\left\langle\Delta E_{c}\right\rangle$ и $\Delta E_{c v}$ изменяются синхронно. Более того, скорость их изменения с ростом концентрации свободных электронов не противоречит данным работы [5].

Таким образом, результаты, полученные в настоящей работе, подтверждают, что в $\mathrm{Bi}_{2} \mathrm{Te}_{3}$ действительно существует подзона „тяжелых“ электронов, и показывают, что обменное взаимодействие свободных электронов может существенным образом осложнить процесс ее выявления при помощи различных экспериментальных методик.

Финансовая поддержка исследований частично получена в рамках проекта РФФИ № 16-08-00478.

\section{Список литературы}

[1] А.Н. Вейс. Изв. вузов. Физика, 7, 50 (2008).

[2] А.Н. Вейс, Л.Н. Лукьянова, В.А. Кутасов. ФТТ, 54 (11), 2051 (2012).

[3] Б.М. Гольцман, В.А. Кудинов, И.А. Смирнов. Полупроводниковые термоэлектрические материалы на основе $\mathrm{Bi}_{2} \mathrm{Te}_{3}$ (М., Наука, 1972) с. 320.

[4] H. Köhler. Phys. Status Solidi B, 73 (1), 95 (1976).

[5] А.Н. Вейс, М.К. Житинская, Л.Н. Лукьянова, В.А. Кутасов. Науч.-техн. ведомости СПбГПУ. Физ.-мат. науки, 3 (177), 29 (2013).

[6] В.Л. Бонч-Бруевич. Вопросы электронной теории сильно легированных полупроводников. Итоги науки (М., ВИНИТИ, 1965) с. 127.

[7] K.H. Unkelbach, C.H. Becker, H. Köhler, A.V. Middendorf. Phys. Status Solidi B, 60 (1), K-41 (1973).

[8] E. Haga, H. Kimura. J. Phys. Soc. Jpn, 19 (9), 1596 (1964).

[9] А.Н. Вейс, М.К. Житинская, Л.Е. Шелимова. Докл. ХІІІ Межгос. сем. „Термоэлектрики и их применения“ (СПб., 2013) с. 144.

Редактор Г.А. Оганесян 


\title{
Conduction band structure of bismuth telluride according to the optical absorption
}

\author{
A.N. Veis ${ }^{1}$, L.N. Lukyanova ${ }^{2}$, V.A. Kutasov ${ }^{2}$ \\ ${ }^{1}$ Peter the Great St. Petersburg Polytechnic University, \\ 195251 St. Petersburg, Russia \\ ${ }^{2}$ loffe Institute, \\ 194021 St. Petersburg, Russia
}

Abstract Spectral dependences of optical absorption coefficient of $n-\mathrm{Bi}_{2} \mathrm{Te}_{3}$ are studied in the range of 40 to $300 \mathrm{meV}$ depending on the electron concentration and the sample thickness at room temperature to determine the parameters of additional subband in the conduction band of bismuth telluride and its impact on charge carrier transport. It is shown that bismuth telluride is a direct bandgap semiconductor with additional subzone in the conduction band, which is consistent with the study of quantum oscillations in $n-\mathrm{Bi}_{2} \mathrm{Te}_{3}$ in strong magnetic fields at temperatures below $20 \mathrm{~K}$. 